\title{
Finite-Volume Statistical Mechanics of Two-Component Coulomb-like Systems and the Principle of Macroscopic Equivalence
}

\author{
Michael K.-H. Kiessling \\ Courant Institute of Mathematical Sciences, New York University, New York, NY 10012, USA \\ Received February 4, 1991, in revised form November 25, 1991
}

\begin{abstract}
Classical stable charge-symmetric two-component systems are discussed in a fixed domain $\Lambda \subset \mathbb{R}^{\mathrm{d}}$. The limit $N \rightarrow \infty$ of the finite- $N$ canonical Gibbs ensemble is compared with the results obtained from a discussion of the Gibbs measures on the space of infinite configurations (the states). A first-order phase transition in the Gibbs states is proved for a large class of interactions, including regularized Coulomb interactions for $d \geqq 3$. In the latter case the transition is isomorphic to an implosion/explosion transition in regularized gravitational systems. Spherical symmetry is not assumed. A transition occurs for certain largedomain/low-temperature pairs $\left(\Lambda, \beta^{-1}\right)$, but ceases to exist in the infinite-volume ensemble. The phase transition supports the conjecture that the standard thermodynamic-limit sequence can be nonuniform even for standard $H$-stable Hamiltonians. The results about the limit of the finite- $N$ ensemble are less complete due to lack of sufficient control of the correlations. However, some notable differences between both descriptions are shown, which are caused by noncommuting limits. Possible physical consequences and open questions are pointed out.
\end{abstract}

\section{Introduction}

For systems with suitably restricted interactions, e.g., stability and temperedness may be required, equilibrium thermodynamics is normally defined in terms of the infinite volume thermodynamic limit. The monographs by Ruelle [Rue 1] and Balescu [Bal], for instance, give an overview over the general ideas and rigorous results. After many remarkable achievements about the existence of the T-limit, e.g., [vHo, Yan-Lee, Lee-Yan, Rue 2, 3, Fis, Gri 1, 2, Lie-Leb, Frö-Par 1, 2], one reasonable next step is to inquire into the fundamental problem of how big a system must be in order to belong to the asymptotic regime of the T-limit. According to the physics folklore, $10^{23} \approx \infty$, emphasizing the laboratory evidence. It seems to be a challenging task to give rigorous estimates for the 
minimum system size that is required for acceptable quantitative agreement of finite systems' properties with their asymptotic limits.

A related but somewhat simpler question is whether any such minimum size is sensitive to the thermodynamic parameters like particle density or temperature. So one may ask whether a T-limit sequence is uniform. If a T-limit sequence is nonuniform, then for any finite system of the sequence there will be a range of thermodynamic parameters for which effects occur which are qualitatively different from what is described by the T-limit.

In the latter case a definition of the thermodynamics on basis of the T-limit would fall short of certain, perhaps important effects. We should not expect anything like this to occur for systems describing normal matter under normal conditions, however, for it would mean a violation of an empirical principle that was discovered for systems of laboratory size. This is the principle of macroscopic equivalence [Bal], which states that the relations between macroscopic quantities like pressure, density and temperature of a substance in equilibrium do not depend on size and shape of a vessel which confines the substance ${ }^{1}$. The principle is a basic building block of standard thermodynamics. It ensures that the systems to which it applies belong to the asymptotic regime of the T-limit.

On the other hand, an example of a nonuniform T-limit sequence is the overall neutral point vortex model; see [Frö-Rue, Cam-O'N]. However, the vortex Hamiltonian is quite peculiar for the finite system's total phase space mass is finite. In this article we inquire rigorously into the possibility of a nonuniformity of the $\mathrm{T}$-limit sequence and a corresponding violation of the principle of macroscopic equivalence for classical systems with standard Hamiltonian. The work is motivated by the observation that in the mean-field approximation some statistical mechanics models indeed behave characteristically different in a finite volume versus in infinite space. In particular, in [Kie 1] the mean-field approximation for two-component systems with certain regularized Coulomb interactions in a ball was shown to give the same equations as the mean-field limit for classical gravitating one-component systems with regularized interactions [Kie 2] does. The mean-field approximation in spherical geometry yields a phase transition which seems to be incompatible with the principle of macroscopic equivalence.

In the present article we shall give the mean-field model of [Kie 1] a rigorous meaning in terms of the Gibbs states on the space of infinite configurations in a finite domain. We will be able to show that the principle of macroscopic equivalence does not apply to these states because of a collapse phase transition. The proof of the transition can be reinterpreted in terms of the mean-field states of classical unstable systems. It seems to be the first proof of this kind that does not assume spherical symmetry.

After discussing the Gibbs states we need inquire into the relation of the Gibbs states with the finite system's measures. It is here where the hard work begins. We will derive several results about the finite- $N$ correlations but will not be able to control them fully. This part of the present paper should be viewed only as a first step toward more complete results.

The problem is the following. For $d \in \mathbb{N}$, let $\Lambda \subset \mathbb{R}^{\mathrm{d}}$ be a (not necessarily simple) connected open domain with volume $|\Lambda|$. The configuration space of a system of $2 N$ classical particles is $\Lambda^{\times 2 N}, N \in \mathbb{N}$. The system's index set $\mathbb{I} \subset \mathbb{N}$

${ }^{1}$ Clearly, this must not be taken literally; one refers to the leading digits of the measured quantities 
contains an equal number of even and odd integers, such that card (II) $\equiv|\mathbb{I}|=2 \mathrm{~N}$. Particle quantities of the one species have even integers as subscript, those of the other odd ones. The location $\mathbf{r}_{i}$ of the $i^{\text {th }}$ particle is a point in the $i^{\text {th }}$ copy of $\Lambda$. Let further $V: \mathbb{R}^{+} \cup\{0\} \rightarrow \mathbb{R}$ be continuous and such that the potential energy $U^{(I)}$ of the system is bounded from below by

$$
U^{(\mathbb{I})}(\mathbf{X})=\frac{1}{2} \sum_{i \in \mathbb{I}} \sum_{j \in \mathbb{I} \backslash\{i\}}(-1)^{i+j} V\left(\left|\mathbf{r}_{i}-\mathbf{r}_{j}\right|\right) \geqq-B N .
$$

Here, $B>0$ is independent of $N$, and $\mathbf{X}$ is a configuration $\left\{\mathbf{r}_{i} \in \Lambda \mid i \in \mathbb{I}\right\}$. For instance, if $V$ is a function of positive type, (1.1) is satisfied with $B=V(0)$ [FisRue; Sect. III]. Presumably (1.1) is true for more general $V$; however, here we take $V$ of positive type. Classical Coulomb systems with regularized singularity are included as special case. Therefore, one may suggestively speak of a "Coulomb system", with "positive" and "negative charges". Let $d X=\prod_{i \in \mathbb{I}} d^{d} r_{i}$. The canonical equilibrium probability measure restricted ${ }^{2}$ to $\Lambda^{\times 2 N}$ is given by

$$
\begin{gathered}
\mu^{(\mathbb{I I})}(d X)=Q^{-1} \exp \left[-\beta U^{(\mathbb{I I})}(\mathbf{X})\right] d X, \\
Q=\int_{\Lambda^{\times 2 N}} \exp \left[-\beta U^{(\mathbb{I})}(\mathbf{X})\right] d X .
\end{gathered}
$$

As usual, $\beta^{-1}=k_{B} T \geqq 0$, where $T$ is the temperature and $k_{B}$ Boltzmann's constant. We will be concerned with the evaluation of the measure (1.2) and its thermodynamic functions in the limit where $\mathbb{I} \rightarrow \mathbb{N}$ with $\Lambda$ fixed, and we will compare the results with those obtained from a study of the Gibbs measures on the infinite configurations. Occasionally we write $\mu^{(N)}$ for $\mu^{(\mathbb{I})}$ to emphasize the cardinality.

The limit envisaged above is an infinite-density limit in a finite volume, in contradistinction to the usual infinite-volume limit at finite density. The limit is suggested by the fact that it keeps the length scale

$$
\lambda_{\text {inh }}=\left(\varepsilon_{0} k_{B} T|\Lambda| / q^{2}\right)^{1 / 2}
$$

fixed, which occurs naturally in the formal mean-field model discussed in [Kie 1]. Here, $\varepsilon_{0}$ is the vacuum permittivity and $q$ the modulus of the electric charge of a particle. Moreover $\Lambda$ needs be close to ball shape. The scale (1.3) measures an inhomogeneity of the one-particle mean-field density. Notice that $\lambda_{\text {inh }} \rightarrow \infty$ as $|\Lambda| \rightarrow \infty$.

An infinite-density limit is not so unusual as it might appear at first sight. Recall that an infinite-density limit in a finite volume is appropriate for the discussion of classical systems with unstable interactions [Mes-Spo, Kie 3], for which either the coupling constant in the interactions is rescaled $\sim N^{-1}$ at fixed temperature or, what is equivalent, the temperature is scaled $\sim N$ at fixed coupling constant. ${ }^{3}$ The novel feature is that our interactions are stable and no scaling of the temperature is required. This brings about some interesting new phenomena, as well as new technical problems.

\footnotetext{
2 The momentum space contributions to the canonical Gibbs measure factor out in the usual way and are discarded here

3 See, however, the article by Compagner [Com] for a scaled finite-volume limit for stable interactions which is equivalent to the T-limit
} 
The space of infinite configurations will be studied first. The Gibbs hypothesis for the mean free-energy functional on the space of infinite configurations defines the infinite configuration Gibbs measures (states). In the next section we show that any state on the space of infinite configurations is necessarily a convex linear combination of product states, i.e., uncorrelated states. It will also be shown that these Gibbs states are locally charge neutral, and that they satisfy the mean-field model that was obtained in [Kie 1] only by a heuristic variational principle. The discussion of this mean-field model, which was started in [Kie 2] in the different context of spherically symmetric gravitational-like systems in $\mathbb{R}^{3}$, will then be considerably expanded in Sect. 3. In particular, a first-order phase transition will be proven without the restriction to spherical symmetry, for a much wider class of interactions [see (R1) and (R2) in Sect. 3], and for all space dimensions $d>2$. It will be shown that the transition ceases to exist in the subsequent limit $|\Lambda| \rightarrow \infty$. Some possible physical applications are outlined at the end of that section.

In subsequent sections we shall study the relation to the finite- $N$ problem in more detail. It is easily shown that the finite- $N$ configurational free energy per particle $-(2 N \beta)^{-1} \log \left(|\Lambda|^{-2 N} Q\right)$ has a limit $f^{*}(\beta, \Lambda)$ as $N \rightarrow \infty$ in $\Lambda$ (Sect. 4). Some of its basic properties are listed, which follow by simple convexity arguments. It will then be shown that $f^{*}$ does not satisfy the Gibbs variational principle for the mean free energy of the infinite configurations, and in Sect. 5 the discrepancy will be located in the (finite- $N$ ) correlations. Although we can prove that any weak limit $\mu$ of (1.2) is an average of locally charge-neutral correlationfree states, the finite- $N$ correlations give a negative contribution to the free energy which has a nonvanishing limit $\phi(\beta)$ as $N \rightarrow \infty$. Thus, for $\mu$ a weak limit of (1.2), $f^{*}=f(\mu)+\phi$, where $f(\mu)$ is the mean free-energy functional at $\mu$, and $\phi<0$ is a temperature-dependent shift. Note that $\phi$ is not a functional of $\mu$. Due to lack of sufficient control of $\phi$, we cannot (yet) prove that the weak limits of (1.2) coincide with the Gibbs states on the space of infinite configurations, as was expected in [Kie 1]. In this sense the validity of Gibbs' hypothesis for $f(\mu)$ has to be postulated in the present work.

\section{Permutation Invariant Probability Measures on Infinite Cartesian Products}

\section{2a. Representation of States}

The space of infinite configurations $\left\{i \mapsto \mathbf{r}_{i} \in \Lambda \mid i \in \mathbb{N}\right\}$ in the infinite Cartesian product $\Lambda^{\mathbb{N}}$ is considered here with the additional constraint that the even integers are mapped to one species, the odd ones to the other. A state on the space of infinite configurations is given by any probability measure on $\Lambda^{\mathbb{N}}$ which is permutation invariant against any finite permutation of indices within each species separately and also invariant against the complete exchange of all even against all odd integers. The space of these charge-symmetric states is denoted $\mathscr{P}\left(\Lambda^{\mathbb{N}}\right)$. It is compact in the weak topology induced by the Borel sets of $\Lambda$, moreover a convex set with invariance group. As such it has an integral representation over the extremal invariant states $\mathscr{E}\left[\mathscr{P}\left(\Lambda^{\mathbb{N}}\right)\right]$. The chargesymmetric states are a subset of the probability measures that are just permutation invariant within even and odd integers separately, denoted here by $\widetilde{\mathscr{P}}\left(\Lambda^{\mathbb{N}}\right)$. 
Proposition 2.1. Denote the even positive integers by $2 \mathbb{N}$ and the odd ones by $2 \mathbb{N}-1$. Then $\mathscr{E}\left[\tilde{\mathscr{P}}\left(\Lambda^{\mathbb{N}}\right)\right]$ consists of all product measures of the type $\varrho_{1}^{\otimes 2 \mathbb{N}} \otimes \varrho_{2}^{\otimes(2 \mathbb{N}-1)}$ with $\varrho_{1,2} \in \mathscr{M}_{+, 1}(\Lambda)$ arbitrary.

Proof. Consider the infinite group of all finite permutations on $\mathbb{N}$, with elements $\pi: \mathbb{N} \ni i \mapsto \pi(i) \in \mathbb{N}$ 1-to-1 onto. The special elements that leave the odd integers unpermuted are abbreviated as $\pi_{e}$. Let further $\xi: 0 \mathbb{N} \times \mathbb{N} \rightarrow \mathbb{N} ;(2 i-1,2 i) \mapsto i$ be the natural neutral pairing. Clearly, ()$\circ \pi_{e}$ means $\left(2 i-1, \pi_{e}[2 i]\right) \mapsto i$. The measures in $\widetilde{\mathcal{P}}\left(\Lambda^{\mathbb{N}}\right)$ form a subspace of every space of probability measures on $\Lambda^{\mathbb{N}}$ that consists of measures which are invariant only under all permutations $\pi$ on the pair-index set induced by $\left(8 \circ \pi_{e}\right.$, for given $\pi_{e}$. Let these spaces of probability measures be denoted by $\widetilde{\mathscr{P}}_{\wp \circ \pi_{e}}\left(\Lambda^{\mathbb{N}}\right)$. Clearly, one can generate all possible pairings by keeping the odd integers fixed and permuting only the even integers over all possibilities. Thus,

$$
\widetilde{\mathscr{P}}\left(\Lambda^{\mathbb{N}}\right) \subseteq \bigcap_{\pi_{e}} \tilde{\mathscr{P}}_{\wp \circ \pi_{e}}\left(\Lambda^{\mathbb{N}}\right) .
$$

The elements of $\tilde{P}_{p \circ \pi_{e}}\left(\Lambda^{\mathbb{N}}\right)$ may be recognized as permutation-invariant probability measures of one-component systems, the "particles" being the $\left(: \circ \pi_{e^{-}}\right.$ generated pairs. Thus $\widetilde{\mathscr{P}}_{\mathfrak{p} \circ \pi_{e}}\left(\Lambda^{\mathbb{N}}\right) \cong \hat{\mathscr{P}}_{\mathfrak{\wp} \circ \pi_{e}}\left([\Lambda \times \Lambda]^{\mathbb{N}}\right)$, where $\widehat{\mathscr{P}}$ means the permutation invariant probability measures for a one-component system. Clearly, the elements of $\widetilde{\mathscr{P}}\left(\Lambda^{\mathbb{N}}\right)$ can be generated from a subset of measures in $\hat{\mathscr{P}}_{\mathfrak{g}} \circ \pi_{e}\left([\Lambda \times \Lambda]^{\mathbb{N}}\right)$ by reinterpretation.

The extreme points of $\hat{\mathscr{P}}_{\mathscr{8} \circ \pi_{e}}\left([\Lambda \times \Lambda]^{\mathbb{N}}\right)$ are known from Theorems 5.1, 5.2, and 5.3 of [Hew-Sav], stating that $\mathscr{E}\left(\hat{\mathscr{P}}_{\mathrm{p}^{\circ} \pi_{e}}\right)$ consists of infinite products of measures in the pair variables, denoted by $\lambda^{\otimes \mathbb{N}}$, where $\lambda \in\left(\mathscr{M}_{+, 1} \otimes \mathscr{M}_{+, 1}\right)(\Lambda \times \Lambda)$. By the above inclusion, each $\mu \in \tilde{\mathscr{P}}\left(\Lambda^{\mathbb{N}}\right)$, interpreted as measure on $[\Lambda \times \Lambda]^{\mathbb{N}}$, is in any $\hat{\mathscr{P}}_{\mathfrak{g} \circ \pi_{e}}\left([\Lambda \times \Lambda]^{\mathbb{N}}\right)$. By the representation theorem of de Finetti, each $\mu \in \widetilde{\mathscr{P}}\left(\Lambda^{\mathbb{N}}\right)$ interpreted on $[\Lambda \times \Lambda]^{\mathbb{N}}$ has a unique integral representation $\mu=\int \tilde{v}_{\mu}(d \lambda) \lambda \otimes \mathbb{N}$, where $\tilde{v}$ is a probability measure on $\left(\mathscr{M}_{+, 1} \otimes \mathscr{M}_{+, 1}\right)(\Lambda \times \Lambda)$, for any arbitrary

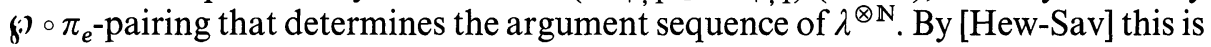
the extremal decomposition.

The decomposition measure $\tilde{v}$ does not depend on the particular choice of $\pi_{e}$. Since $\mu$ is also invariant against permutations of $\xi \circ \pi_{e}^{\prime}$-generated pairs, with $\pi_{e} \neq \pi_{e}^{\prime}$, it follows that $\tilde{v}$ is concentrated on those $\lambda^{\otimes \mathbb{N}}$ which are invariant against any transformation $\pi_{e} \rightarrow \pi_{e}^{\prime}$. The only elements of the form $\lambda^{\otimes \mathbb{N}}$ that satisfy these invariance requirements are infinite products of one-particle measures of the form $\left(\varrho_{1} \otimes \varrho_{2}\right)^{\otimes \mathbb{N}}$ on $(\Lambda \times \Lambda)^{\mathbb{N}}$. Let inj be the natural injection; i.e., inj: $\mathscr{\mathscr { P }}_{\mathfrak{g}} \circ \pi_{e}([\Lambda \times \Lambda])^{\mathbb{N}} \rightarrow \mathscr{\mathscr { P }}_{\mathfrak{g} \circ \pi_{e}}\left(\Lambda^{\mathbb{N}}\right)$. Then inj $\left[\left(\varrho_{1} \otimes \varrho_{2}\right)^{\otimes \mathbb{N}}\right]=\varrho_{1}^{\otimes 2 \mathbb{N}} \otimes \varrho_{2}^{\otimes(2 \mathbb{N}-1)}$.

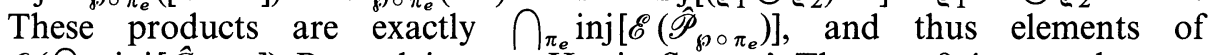
$\mathscr{E}\left(\bigcap_{\pi_{e}}\right.$ inj $\left.\left[\hat{\mathscr{P}}_{g} \pi_{e}\right]\right)$. By applying now Hewitt-Savage's Theorem 9.4 we see that any element of $\mathscr{\mathscr { P }}\left(\Lambda^{\mathbb{N}}\right)$ has a unique integral representation over the products $\varrho_{1}^{\otimes 2 \mathbb{N}} \otimes \varrho_{2}^{\otimes(2 \mathbb{N}-1)}$, which are extreme points of $\mathscr{P}\left(\Lambda^{\mathbb{N}}\right)$, thus all of $\mathscr{E}\left[\widetilde{\mathscr{P}}\left(\Lambda^{\mathbb{N}}\right)\right]$.

By Proposition 2.1, any $\mu$ in $\mathscr{P}\left(\Lambda^{\mathbb{N}}\right)$ can be uniquely represented in the form

$$
\mu=\int \tilde{v}_{\mu}\left(d \varrho_{1} \otimes d \varrho_{2}\right) \varrho_{1}^{\otimes 2 \mathbb{N}} \otimes \varrho_{2}^{\otimes(2 N-1)}
$$

with $\tilde{v}_{\mu}$ concentrated on $\mathscr{M}_{+, 1}^{\otimes 2}(\Lambda \times \Lambda)$ and satisfying the symmetry condition

$$
\int \tilde{v}_{\mu}\left(d \varrho_{1} \otimes d \varrho_{2}\right) \varrho_{1}^{\otimes 2 \mathbb{N}} \otimes \varrho_{2}^{\otimes(2 \mathbb{N}-1)}=\int \tilde{v}_{\mu}\left(d \varrho_{1} \otimes d \varrho_{2}\right) \varrho_{2}^{\otimes 2 \mathbb{N}} \otimes \varrho_{1}^{\otimes(2 \mathbb{N}-1)} \text {. }
$$


This guarantees the symmetry under exchange of both species.

It should be noticed that (2.1) is in general not the extremal decomposition on $\mathscr{P}\left(\Lambda^{\mathbb{N}}\right)$ but on $\tilde{\mathscr{P}}\left(\Lambda^{\mathbb{N}}\right)$. We shall see in a moment, however, that the Gibbs states of the Coulomb-like systems are averages over the $\varrho^{\otimes \mathbb{N}}$ which are extremal states of $\mathscr{P}\left(\Lambda^{\mathbb{N}}\right)$.

\section{2b. Thermodynamic Functions for the States}

For $\mu \in \mathscr{P}\left(\Lambda^{\mathbb{N}}\right)$, a marginal measure $\mu_{m, q}$ of $\mu$ is defined by specifying its total number of coordinates $m=n_{+}+n_{-}$and its charge number $q=n_{+}-n_{-}$.

Let $\mathbf{N} \subset \mathbb{N}$ contain an equal number of odd and even integers, such that $\operatorname{card}(\mathbf{N})=|\mathbf{N}|=2 n$. Given $\mu \in \mathscr{P}\left(\Lambda^{\mathbb{N}}\right)$, the $\mu$-entropy $S_{\mu}(\mathbf{N})$ of the set $\mathbf{N}$ is defined in terms of the neutral marginal $\mu_{2 n \mid 0}$ as

$$
S_{\mu}(\mathbf{N})=-\infty ; \quad \mu_{2 n \mid 0} \text { is singular }
$$

and

$$
S_{\mu}(\mathbf{N})=-k_{B} \int_{\Lambda^{\times 2 n}} \eta_{2 n \mid 0} \log \left(|\Lambda|^{2 n} \eta_{2 n \mid 0}\right) \prod_{i \in \mathbf{N}} d^{d} r_{i}
$$

if $d \mu_{2 n \mid 0} \ll d^{d 2 n} r$. In (2.3b), $\eta_{2 n \mid 0}$ is the density of $\mu_{2 n \mid 0}$, with variables indexed by $\mathbf{N}$. Permutation invariance of $\mu$ gives $S_{\mu}(\mathbf{N})=S_{\mu}\left(\mathbf{N}^{*}\right)$ for all neutral sets $\mathbf{N}, \mathbf{N}^{*}$ which have the same cardinality. The $\mu$ entropy has the following important properties:

\section{Proposition 2.2.}

Non-positivity of $S_{\mu}(\mathbf{N})$ :

$$
S_{\mu}(\mathbf{N}) \leqq 0 .
$$

Monotonic decrease of $S_{\mu}(\mathbf{N})$ : If $|\mathbf{N}| \leqq\left|\mathbf{N}^{\prime}\right|$, then

$$
S_{\mu}\left(\mathbf{N}^{\prime}\right) \leqq S_{\mu}(\mathbf{N}) .
$$

Strong sub-additivity of $S_{\mu}(\mathbf{N})$ :

$$
S_{\mu}\left(\mathbf{N}^{\prime} \cup \mathbf{N}^{\prime \prime}\right)+S_{\mu}\left(\mathbf{N}^{\prime} \cap \mathbf{N}^{\prime \prime}\right) \leqq S_{\mu}\left(\mathbf{N}^{\prime}\right)+S_{\mu}\left(\mathbf{N}^{\prime \prime}\right) .
$$

Proof. For the proof of Proposition 2.2 the reader may wish to reformulate Sect. 2 of ref. [Rob-Rue] (proof of Proposition 1) for the finite-volume case studied here. A proof based entirely on Jensen's inequality is given in [Kie 3].

This allows us to define for each $\mu$ the mean entropy $s(\mu)$ via

\section{Definition 1.}

$$
s(\mu) \equiv \lim _{\mathbf{N} \rightarrow \mathbb{N}}|\mathbf{N}|^{-1} S_{\mu}(\mathbf{N})
$$

with $\operatorname{ran}(s)=[-\infty, 0]$.

Remark. The limit is well defined and yields $s(\mu)=\inf _{\mathbf{N}}|\mathbf{N}|^{-1} S_{\mu}(\mathbf{N})$. See, e.g., Lemma IX.2.4 of Ref.[Ell].

Proposition 2.3. The mean entropy is affine upper semi-continuous on $\mathscr{P}\left(\Lambda^{\mathbb{N}}\right)$. 
Proof. The proof that $s(\mu)$ is affine is elementary. The proof of upper semicontinuity proceeds by noting that $s(\mu)$ is the lower limit of upper semicontinuous functions, all of which are lower limits of some sequence of continuous functions. As such one can construct a sequence of continuous functions of which $s(\mu)$ is the lower limit, hence $s(\mu)$ is upper semi-continuous. See the corresponding proof in [Rob-Rue].

Since $s(\mu)$ is affine, by (2.1) we find

$$
s(\mu)=\int \tilde{v}_{\mu}\left(d \varrho_{1} \otimes d \varrho_{2}\right) \frac{1}{2}\left[\mathscr{S}\left(\varrho_{1}\right)+\mathscr{S}\left(\varrho_{2}\right)\right]
$$

with $\mathscr{S}(\varrho)=-k_{B} \int_{\Lambda} \rho \log (|\Lambda| \rho) d^{d} r$ for $\varrho\left(d^{d} r\right)=\rho(\mathbf{r}) d^{d} r$, and $-\infty$ else.

The energy of a set of particles $\mathbf{N}$ consists of two contributions, the one that comes from the interactions among the $2 n$ particles in $\mathbf{N}$ and the one that comes from the interactions of the particles in $\mathbf{N}$ with the other particles of the infinite system represented by $\mu$. It must be defined in terms of a limiting procedure. Let $\mathbf{M} \cap \mathbf{N}=\emptyset$, with $|\mathbf{M}|=2 m$. Then

$$
E_{\mu}(\mathbf{N})=I_{\mu}(\mathbf{N})+\lim _{\mathbf{M} \rightarrow \mathbf{N} \backslash \mathbf{N}} C_{\mu}(\mathbf{N} \mid \mathbf{M})
$$

with the internal energy

$$
I_{\mu}(\mathbf{N})=\int_{\Lambda^{\times 2 n}} \sum_{i<j \in \mathbf{N}}(-1)^{i+j} V\left(\left|\mathbf{r}_{i}-\mathbf{r}_{j}\right|\right) \mu_{2 n \mid 0}\left(\prod_{i \in \mathbf{N}} d^{d} r_{i}\right)
$$

and the coupling energy

$$
\begin{aligned}
C_{\mu}(\mathbf{N} \mid \mathbf{M})= & \frac{1}{2} \int_{A^{\times 2(n+m)}} \sum_{i \in \mathbf{N}} \sum_{j \in \mathbf{M}}(-1)^{i+j} V\left(\left|\mathbf{r}_{i}-\mathbf{r}_{j}\right|\right) \\
& \times \mu_{2(n+m) \mid 0}\left(\prod_{i \in \mathbf{N}} \prod_{j \in \mathbf{M}} d^{d} r_{i} d^{d} r_{j}\right) .
\end{aligned}
$$

The factor $1 / 2$ in $(2.5 \mathrm{c})$ comes from splitting the coupling energy evenly between the system $\mathbf{N}$ and the system $\mathbf{M}$.

Using the representation (2.1) for $\mu,(2.5)$ is readily rewritten into a superposition of bilinear forms involving the one-particle measures $\varrho_{1}$ and $\varrho_{2}$. By the symmetry condition (2.2) one finds that $C_{\mu}(\mathbf{N} \mid \mathbf{M})$ is an average over terms of the form

$$
n m \frac{1}{2} \int_{\Lambda^{\times 2}} V\left(\left|\mathbf{r}-\mathbf{r}^{\prime}\right|\right)\left(\varrho_{1}-\varrho_{2}\right)^{\otimes 2}\left(d^{d} r d^{d} r^{\prime}\right) .
$$

Since $V$ is of positive type, each term of the form (2.6) diverges to $+\infty$ as $m \rightarrow \infty$, unless the decomposition measure $\tilde{v}$ is concentrated on those $\varrho_{1} \otimes \varrho_{2}$ which satisfy $\varrho_{1} \equiv \varrho_{2}$. In the latter case we may call $\mu$ locally charge neutral. For a locally charge neutral $\mu$ the contribution $C_{\mu}$ to the energy of $\mathbf{N}$ vanishes. Rewriting $\mathbf{I}_{\mu}$ in terms of the marginal $\mu_{2 \mid 0}$, we get

$$
E_{\mu}(\mathbf{N})=I_{\mu}(\mathbf{N})=-n \int_{A^{2}} V\left(\left|\mathbf{r}-\mathbf{r}^{\prime}\right|\right) \mu_{2 \mid 0}\left(d^{d} r d^{d} r^{\prime}\right)
$$

for a locally charge neutral state. 
Since $(1 / 2 n) E_{\mu}(\mathbf{N})$ is thus either independent of $n$ and finite or else $+\infty$, a mean ideal energy of $\mu$ can now be defined by

\section{Definition 2.}

$$
e(\mu) \equiv-\frac{1}{2} \int_{\Lambda^{2}} V\left(\left|\mathbf{r}-\mathbf{r}^{\prime}\right|\right) \mu_{2 \mid 0}\left(d^{d} r d^{d} r^{\prime}\right)
$$

if $\tilde{v}_{\mu}($.$) is concentrated on products of the form \varrho^{\otimes 2}$, and $e(\mu)=+\infty$ else.

For all $\mu$ with a decomposition measure concentrated on the charge-symmetric product measures, the representation (2.1) gives

$$
e(\mu)=\int v_{\mu}(d \varrho) e\left(\varrho^{\otimes \mathbb{N}}\right)=-\int v_{\mu}(d \varrho) \frac{1}{2} \int_{\Lambda^{2}} V\left(\left|\mathbf{r}-\mathbf{r}^{\prime}\right|\right) \varrho^{\otimes 2}\left(d^{d} r d^{d} r^{\prime}\right)
$$

with the measure $v_{\mu}$ living on $\mathscr{M}_{+, 1}(\Lambda)$. Clearly, this is a superposition of meanfield energies, which is characteristic of ideal systems [Bal].

From (2.3) and (2.7) one can construct an ideal $\mu$ free energy for a set $\mathbf{N}$ via

$$
F_{\mu}(\mathbf{N})=E_{\mu}(\mathbf{N})-T S_{\mu}(\mathbf{N}) \text {. }
$$

For $e(\mu)<\infty$ the corresponding mean ideal free energy of $\mu$ is given by

\section{Definition 3.}

$$
f(\mu) \equiv e(\mu)-T s(\mu)
$$

and $f(\mu)=\infty$ else.

Proposition 2.3 implies that the mean ideal free-energy functional $f(\mu)$ is affine lower semi-continuous on $\mathscr{P}\left(\Lambda^{\mathbb{N}}\right)$. This means that $f(\mu)$ takes its infimum on a subset of $\mathscr{E}\left[\mathscr{P}\left(\Lambda^{\mathbb{N}}\right)\right]$. Moreover, since $f(\mu)$ is affine, if $\mu$ has a decomposition measure concentrated on the charge-symmetric product measures $\varrho^{\otimes \mathbb{N}}$ one has the useful representation

$$
f(\mu)=\int v_{\mu}(d \varrho) f\left(\varrho^{\otimes \mathbb{N}}\right)=\int v_{\mu}(d \varrho) \mathscr{F}(\rho)
$$

or else $f(\mu)=+\infty$. The functional $\mathscr{F}$ is defined by (2.10) and reads

$$
\mathscr{F}(\rho)=-\frac{1}{2} \int_{\Lambda} \int_{\Lambda} \rho(\mathbf{r}) \rho\left(\mathbf{r}^{\prime}\right) V\left(|\mathbf{r}-\mathbf{r}|^{\prime}\right) d^{d} r d^{d} r^{\prime}+k_{B} \int_{\Lambda} \rho \log (|\Lambda| \rho) d^{d} r
$$

for $d \varrho \ll d^{d} r$, and $\mathscr{F}=\infty$ else. From (2.10) it follows that any global minimizer $\mu^{*}$ of $f(\mu)$ has its decomposition measure $v_{\mu^{*}}(d \varrho)$ concentrated on the set $\mathscr{M}_{+, 1}^{*}(\Lambda)$ of global minimizers of $\mathscr{F}(\rho)$. The infimum is finite, for $0 \geqq e(\mu) \geqq-(1 / 2) V(0)$ and the negativity of $s(\mu)$ imply that the functional $f(\mu)$ is bounded below. Furthermore, any global minimizer is a solution of

$$
\rho=\exp (\beta V * \rho) / \int_{A} \exp (\beta V * \rho) d^{d} r .
$$

The Gibbs' hypothesis for the ideal mean free energy of states $\mu \in \mathscr{P}\left(\Lambda^{\mathbb{N}}\right)$ naturally leads to the mean-field picture anticipated in [Kie 1]. 


\section{Phase Transitions of Gibbs States and the Principle of Macroscopic Equivalence}

In the present section we study the qualitative behavior of the Gibbs states on the infinite configurations. Notice that the mean free-energy functional (2.11) is identical in structure to the corresponding functional constructed in the meanfield limit for unstable systems. Unstable one-component systems have been studied analytically in various classical [Thi, Mes-Spo, Kie 2,3] and quantum systems [Her-Thi 1, H-N-T, Pfl, Mes 1-4]. For numerical evaluations see [HerThi 2].

This section also improves considerably on the results obtained in [Kie 2]. A broader class of interactions is covered; a phase transition is now proven in all space dimensions $d>2$; spherical symmetry is not assumed; some regularity results state piecewise $C^{\infty}$ instead of the previous $C^{2}$ for certain thermodynamic functions.

Let $R \mapsto \Lambda(R)$ be an increasing sequence of open domains $\subset \mathbb{R}^{d}$, such that for each $R$ there exist open $d$-dimensional balls $B_{R}$ and $B_{R^{\prime}}$ with $B_{R^{\prime}} \subset \Lambda(R)$ $\subset B_{R}$, with fixed ratio of the radii, $R^{\prime} / R=h \in(0,1]$. By translation invariance of the problem, we may assume that $B_{R}$ is always centered at the origin.

The interaction potential $V(r)$ will now be subject to the following additional requirements:

$$
0 \leqq V(r) \leqq \kappa r^{-d+2} \equiv V_{C}(r)
$$

for all $r \leqq R$, with $d^{*}=d-2$, and with $\kappa^{-1}=q^{-2} \varepsilon_{0}^{(d)} d^{*}\left|S^{d-1}\right|$.

$$
V(r) \geqq V\left(2 a_{0}\right) ; \quad r \leqq 2 a_{0} .
$$

Here, $a_{0}$ is a typical microscopic length. The charge $q$ and the dielectric constant for $d$-dimensional space, $\varepsilon_{0}^{(d)}$, are included merely for convenience. This is to facilitate the quantitative interpretation of the results in the special but physically important case of Coulomb systems in three space dimensions, where we have $\varepsilon_{0}^{(3)}=\varepsilon_{0}$. This special case was discussed under more restrictive assumptions in [Kie 1].

Given these hypotheses, the existence of a phase transition for pairs $[\beta, \Lambda(R)]$ can be proved in two main steps. The first one is to show that there are hightemperature states, the free energy of which is bounded below independently of $R$.

Proposition 3.1. For $\Lambda=\Lambda(R)$ there exists a $\beta_{*}(R)$, with $\beta_{*}(R) \rightarrow \infty$ for $R \rightarrow \infty$, and a unique right- $C^{\infty}$ mapping of $\left[0, \beta_{*}\right)$ into a subset $\left\{\rho^{(I)}(\beta)\right\}$ of the local minimizers of $\mathscr{F}(\rho)$, with the special case $0 \mapsto|\Lambda|^{-1}$. Moreover there exists $a \widehat{\beta}>0$ which depends on $\sup _{r} V$ but not on $\Lambda$, and for each $\beta \in[0, \widehat{\beta})$ there exists a unique global minimizer of $\mathscr{F}(\rho)$ such that the local minimizers $\rho^{(I)}$ are global for $\beta<\breve{\beta}$. The corresponding mapping $\beta \mapsto\left[\mathscr{F}\left(\rho^{(I)}\right)\right](\beta)$ is right $-C^{\infty}$ on $\left[0, \beta_{*}\right)$, and it is bounded below by a bound $\mathscr{F}_{*}(\beta)$ that is independent of $a_{0}$ and $R$.

We see that eventually $[0, \breve{\beta}) \subset\left[0, \beta_{*}\right)$, and the densities $\rho^{(I)}$ need not be global minimizers on $\left(\not \beta, \beta_{*}\right)$. Indeed, the second step is to prove that for $R$ and $\beta$ big enough, with $\beta<\beta_{*}$, there are other states, of type $I I$, say, which yield a free energy $\mathscr{F}\left(\rho^{(I I)}\right)$ that is lower than $\mathscr{F}\left(\rho^{(I)}\right)$.

Proposition 3.2. Let the ball $B_{a_{0}}$ be contained in $\Lambda$. By $\rho_{0}$ denote the probability density which is homogeneously concentrated on $B_{a_{0}}$. Then there exists a $R_{*}$ such that for $R>R_{*}$ there exists a $\hat{\beta} \in\left(\widetilde{\beta}, \beta_{*}\right)$ such that $\mathscr{F}\left(\rho_{0}\right)<\mathscr{F}_{*}$ for $\beta>\hat{\beta}$. 
This implies that, if the volume is big enough, for a huge class of interactions there is a transition between two mappings $\beta \mapsto\left[\rho^{(I)}(\mathbf{r})\right](\beta)$ and $\beta \mapsto\left[\rho^{(I I)}(\mathbf{r})\right](\beta)$ which generate different types of global minimizers of $\mathscr{F}$. The transition must take place at a $\beta_{\mathfrak{1}} \in[\widetilde{\beta}, \hat{\beta})$. The next proposition shows that this transition corresponds to a jump in the derivative of the mapping $\beta \mapsto\left[\inf _{e} \mathscr{F}(\rho)\right](\beta)$.

Proposition 3.3. Let $\beta_{\uparrow}$ be the value of $\beta$ for which a phase transition occurs between a global minimizer $\rho^{(1)}$ of the high-temperature regime and at least one different global minimizer, say $\rho^{(I I)}$, of $\mathscr{F}(\rho)$. Then the left derivative of $\beta \mapsto\left[\inf _{\varrho} \mathscr{F}(\rho)\right](\beta)$ at $\beta=\beta_{\downarrow}$ is strictly greater than its right derivative.

According to Ehrenfest's classification the transition is of first order. Notice that, since the densities of type $\rho^{(I)}$ are at least local minimizers of $\mathscr{F}(\rho)$, a bifurcation is excluded. Generically this rules out a phase transition of order 2 or higher.

To simplify notation, we will write $\int_{A} f$ for $\int_{A} f d^{d} r$ etc.

Proof of Proposition 3.1. The global minimizers of $\mathscr{F}$ are solutions of the EulerLagrange equation (2.12), which is equivalent to

$$
\Psi=\beta V * e^{\Psi} / \int_{\Lambda} e^{\Psi}
$$

with $\Psi=\beta V * \rho$, and $*$ means convolution via integration over $\Lambda$. Given a solution $\Psi$, the corresponding density $\rho$ obviously is given by $e^{\Psi} / \int_{A} e^{\psi}$. Solution properties of this equation for $\Psi$ are conveniently estimated with the help of the comparison problem

$$
\Phi=\beta V_{C} \bullet e^{\Phi} / \int_{B_{R}} e^{\Phi}
$$

with $V_{C}$ defined in (R1), and $\bullet$ means convolution via integration over $B_{R}$. Let us refer to these equations for $\Psi$ and $\Phi$ just by (psi) and (phi), respectively.

For $B_{R}$ a ball of radius $R$ in $d>2$, and for $\beta=\beta^{(s)}$, with

$$
\beta^{(s)} \equiv 2 \varepsilon_{0}^{(d)} q^{-2} R^{d^{*}}\left|S^{d-1}\right|,
$$

(phi) admits the weak solution

$$
\Phi^{(s)}(r)=-2 \log (r / R)+2 / d^{*}
$$

for which $e^{\Phi(s)}$ is in all $L^{p}(\Lambda)$ with $p<d / 2$. Define $\eta^{(s)}=\beta^{(s)} / \int_{B_{R}} \exp \left(\Phi^{(s)}\right)$. Then, for any $\eta \leqq \eta^{(s)}$, the iterational sequence

$$
\begin{aligned}
\chi^{(n+1)} & =\eta V * \exp \left(\chi^{(n)}\right), \\
\chi^{(0)} & =\Phi^{(s)}
\end{aligned}
$$

converges monotonically decreasing to a positive solution of the equation $\chi=\eta V * \exp (\chi)$, referred to by (chi) from now on. This follows from $\eta \leqq \eta^{(s)}$, $\Lambda \subset B_{R}$, (R1), and from monotonicity of exp. In particular, for every such $\eta$ there exists a pointwise minimal positive solution $\hat{\chi}_{\eta}$ of (chi). It is obtained as limit of the monotonically increasing iteration that starts with $\chi^{(0)} \equiv 0$ instead of $\Phi^{(s)}$. The mapping $\eta \mapsto \hat{\chi}_{\eta}$ is (obviously) pointwise monotonically increasing. It is also arbitrarily right-differentiable on $\left[0, \eta^{(s)}\right)$. This follows from the implicit func- 
tion theorem together with a stability argument [Ama]. The mapping $\eta \mapsto B(\eta) \equiv \eta \int_{A} \exp \left(\hat{\chi}_{\eta}\right)$ is thus also right- $C^{\infty}$ and monotonically increasing on $\left[0, \eta^{(s)}\right)$, hence $B$ has a unique inverse $B^{-1}$. Abbreviate $B\left(\eta^{(s)}\right)$ by $\beta_{*}$. (Note that $\beta_{*} \neq \beta^{(s)}$. See also below.) For $\beta \in\left[0, \beta_{*}\right]$, the mapping $\beta \mapsto \hat{\Psi}_{\beta}^{*} \equiv \hat{\chi}_{B^{-1}(\beta)}$ is pointwise monotonically increasing, furthermore right- $C^{\infty}$ on the corresponding open interval, and it generates uniquely solutions of (psi). These solutions of (psi) now uniquely define solutions $\hat{\rho}_{\beta} \equiv \exp \left(\hat{\Psi}_{\beta}\right) / \int_{A} \exp \left(\hat{\Psi}_{\beta}\right)$ of $(2.12)$. Obviously we have $0 \mapsto \hat{\Psi}_{0}=0$, which corresponds to $0 \mapsto \hat{\rho}_{0}=|\Lambda|^{-1}$.

The mapping $\beta \mapsto \hat{\rho}_{\beta}$ goes into the local minimizers of $\mathscr{F}(\rho)$. This is proven just in the same manner as in [Kie 2, Sect.4], there for the special case $d=3$.

For $\beta<\breve{\beta} \equiv 1 / 2 V(0)$, the solutions of (2.12) are unique by [Mes-Spo, Theorem 3]. So for any $\beta<\not{\beta}$ the solution of (2.12) is also the global minimizer of $\mathscr{F}$. The ordering $\beta<\beta_{*}$ for $R$ big enough follows from $\beta_{*}(R) \rightarrow \infty$ for $R \rightarrow \infty$, which is shown as follows. We have $\beta_{*}=c(R) \beta^{(s)}$, with $\beta^{(s)} \sim R^{d-2}$ (see above), and with

$$
c(\mathrm{R})=\int_{A} \exp \left(\hat{\chi}_{\eta}(s)\right) / \int_{B_{R}} \exp \left(\Phi^{(s)}\right) \geqq h^{d}\left(d^{*} / d\right) \exp \left(-2 / d^{*}\right)
$$

which follows from

$$
\int_{\Lambda} \exp \left(\hat{\chi}_{\eta^{(s)}}\right) \geqq\left|S^{d-1}\right|(h R)^{d} / d
$$

[recall that $\hat{\chi}>0$ and $B_{R^{\prime}} \subset \Lambda(R)$ ], and from

$$
\int_{B_{R}} \exp \left(\Phi^{(s)}\right)=\left|S^{d-1}\right| R^{d} \exp \left(2 / d^{*}\right) / d^{*} .
$$

Consequently, on $[0, \breve{\beta})$ the mapping $\beta \mapsto \hat{\rho}_{\beta}$ generates the global minimizers. The densities $\hat{\rho}_{\beta}(\mathbf{r})$ will be called to be of type $I$, and they get the symbol $\left[\rho^{(I)}(\mathbf{r})\right](\beta)$.

The free-energy mapping $\beta \mapsto \mathscr{F}\left(\rho^{(I)}\right)$ is readily shown to be bounded below on $\left(0, \beta_{*}\right)$ by a bound that is independent of $a_{0}$ and $R$. Using (2.12), one shows that $\mathscr{F}(\rho)=\tilde{\mathscr{F}}(\rho)$ for solutions of $(2.12)$, with

$$
\begin{aligned}
\tilde{\mathscr{F}}(\rho)= & \frac{1}{2} \int_{\Lambda} \int_{\Lambda} \rho(\mathbf{r}) \rho\left(\mathbf{r}^{\prime}\right) V\left(\left|\mathbf{r}-\mathbf{r}^{\prime}\right|\right) d^{d} r d^{d} r^{\prime} \\
& -\beta^{-1} \log \left\{|\Lambda|^{-1} \int_{\Lambda} \exp (\beta V * \rho) d^{d} r\right\} .
\end{aligned}
$$

[The identity between the two free-energy functionals is generically true for solutions of (2.12), and false for general $\rho$.] The integral for the interaction energy is positive, since $V$ is positive. To get a lower bound it can be dropped. This and the equivalence of (2.12) with (psi), monotonicity of $\beta \mapsto \Psi_{\beta}$, and finally $\hat{\Psi}_{\beta_{*}}=\hat{\chi}_{B^{-1}\left(\beta_{*}\right)}=\hat{\chi}_{\eta^{(s)}}<\Phi^{(s)}$ yield

$$
\begin{aligned}
\mathscr{F}\left(\rho^{(I)}\right) & -\beta^{-1} \log |\Lambda|>-\beta^{-1} \log \int_{\Lambda} \exp \left(\hat{\Psi}_{\beta}\right) \\
& >-\beta^{-1} \log \int_{\Lambda} \exp \left(\hat{\Psi}_{\beta_{*}}\right)>-\beta^{-1} \log \int_{B_{R}} \exp \left(\Phi^{(s)}\right) \\
& =-\beta^{-1}\left[2 / d^{*}+\log \left(\left|B_{R}\right| d / d^{*}\right)\right] .
\end{aligned}
$$


With $B_{R^{\prime}} \subset A(R) \subset B_{R}$ and $R^{\prime} / R=h$ for all $R$ we find

$$
\mathscr{F}\left(\rho^{(I)}\right)>-\beta^{-1}\left[2 / d^{*}+\log \left(d / d^{*} h^{d}\right)\right] \equiv \mathscr{F}_{*}(\beta)
$$

for all $\beta \leqq \beta_{*}$.

This establishes the existence of a high-temperature phase. The solution branch of (2.12) whose existence has just been proven carries the global minimizers of $\mathscr{F}$ at high enough temperatures, up to infinity.

Proof of Proposition 3.2. By (R2),

$$
\int_{\Lambda} \int_{\Lambda} \rho_{0}(\mathbf{r}) \rho_{0}\left(\mathbf{r}^{\prime}\right) V\left(\left|\mathbf{r}-\mathbf{r}^{\prime}\right|\right) d^{d} r d^{d} r^{\prime} \geqq V\left(2 a_{0}\right) .
$$

Furthermore,

$$
\beta^{-1} \int_{\Lambda} \rho_{0} \log \left(|\Lambda| \rho_{0}\right) d^{d} r \leqq-\beta^{-1} d \log \left(a_{0} / R\right)
$$

by direct calculation. Consider $\mathscr{F}\left(\rho_{0}\right)$ and $\mathscr{F}_{*}$ as functions of all $\beta \in \mathbb{R}^{+}$. Thus one finds that $\mathscr{F}\left(\rho_{0}\right) \leqq \mathscr{F}_{*}$ if $\beta>\hat{\beta}$, with

$$
\hat{\beta}(R)=\frac{2}{V\left(2 a_{0}\right)}\left[\frac{2}{d^{*}}+\log \frac{R^{d} d}{\left(a_{0} h\right)^{d} d^{*}}\right] .
$$

To conclude the existence of a phase transition $\hat{\beta}$ must be smaller than $\beta_{*}$. Indeed, since $\beta_{*} \geqq c R^{d-2}$ (see above) and $\hat{\beta} \sim d \log \left(R / a_{0}\right)$ asymptotically, there exists $R_{*}$ such that $\hat{\beta}<\beta_{*}$ for $R>R_{*}$.

Propositions 3.1 and 3.2 imply now the existence of $\beta_{\ddagger}$ for which at least two different global minimizers of $\mathscr{F}$ exist, provided $R$ is big enough. This means a phase transition at $\beta_{1}$ for all positive bounded interactions of positive type that satisfy (R1) and (R2).

In the following it is shown that the transition proved in Propositions 3.1 and 3.2 is associated with a jump in the potential energy per particle

$$
\mathscr{E}(\rho)=-\frac{1}{2} \int_{\Lambda} \int_{\Lambda} \rho(\mathbf{r}) \rho\left(\mathbf{r}^{\prime}\right) V\left(\left|\mathbf{r}-\mathbf{r}^{\prime}\right|\right) d^{d} r d^{d} r^{\prime}
$$

and in the entropy per particle

$$
\mathscr{S}(\rho)=-k_{B} \int_{\Lambda} \rho \log (|\Lambda| \rho) d^{d} r .
$$

Provided the derivative of the mapping $\beta \mapsto \beta\left[\inf _{\varrho} \mathscr{F}(\rho)\right](\beta)$ exists, it gives the potential energy per particle. Hence, the jump in energy and entropy means a discontinuity in the derivative of the free energy.

Proof of Proposition 3.3. Consider the functional $\tilde{\mathscr{F}}$ given in the proof of Proposition 3.1. Recall that for solutions $\rho$ of $(2.12)$ we have $\tilde{\mathscr{F}}(\rho)=\mathscr{F}(\rho)$. Now assume the energy of the global minimizers depends continuously on $\beta$. Then, since the free energies of both minimizers at the phase transition are equal, it follows from inspection of $\tilde{\mathscr{F}}$ that

$$
\int_{\Lambda} \exp \left(\beta_{\downarrow} V * \rho^{(I)}\right)=\int_{\Lambda} \exp \left(\beta_{\downarrow} V * \rho^{(I I)}\right),
$$


where $\rho^{(I / I I)}$ are now solutions of (2.12) at $\beta_{\downarrow}$. This implies that $\beta_{\downarrow} V * \rho^{(I)}$ and $\beta_{\uparrow} V * \rho^{(I I)}$ are solutions of the auxiliary equation (chi) for the same value of $\eta$. But since $V * \rho^{(I)}$ is pointwise smaller (as minimal solution for this $\eta$ ) than $V * \rho^{(I)}$ (see the proof of Proposition 3.1), this gives a contradiction.

Therefore the energy and the entropy, and thus the derivative of inf $\mathscr{F}$, all must be discontinuous. Since the energy is always a decreasing function of $\beta$ (a general fact), further it follows that $\mathscr{E}\left(\rho^{(I)}\right)>\mathscr{E}\left(\rho^{(I I)}\right)$, where $\mathscr{E}\left(\rho^{(I / I I)}\right)$ is the left/right derivative of $\beta \inf \mathscr{F}$ at $\beta_{\mathfrak{1}}$.

Remark 1. The condition (R1) can obviously be relaxed to $0 \leqq V<C r^{-d+2}$ for any positive $C$. Presumably it can be relaxed to $|V(\mathbf{r})|<C r^{-d+2}$.

Remark 2. The proof obviously fails for 2-dimensional systems. For certain nonCoulomb interactions in two space dimensions one can argue in favor of a finitevolume phase transition of first order just by noting the identity of the mathematical problem to the corresponding one for one-component unstable 2-D systems. Dynamical computer simulations indicating phase transitions for such systems have been studied recently in [C-B-R], [P-N-T].

Because of the jump in the energy we call the new phase below the hightemperature regime the collapsed phase.

The phase transition disappears when one passes over to the infinite-density infinite-volume ensemble. The appropriate objects to study are now the measures $|\Lambda| \rho$. If $\Lambda(R)$ is some increasing sequence of domains which converges to $\mathbb{R}^{d}$ any $\Lambda^{\prime}$ will eventually be contained in $\Lambda(R)$. On any fixed $\Lambda^{\prime}$ we have

Lemma 3.1. For $\beta<\infty$ any equilibrium density $|\Lambda| \rho$ converges pointwise to the constant value 1 as $R \rightarrow \infty$.

Proof. Let $|\Lambda| \mapsto|\Lambda| \rho(|\Lambda|)$ be any sequence of solutions of the family of equations (2.12) (viewing $\Lambda$ as a parameter), with $\beta$ given. Taking the convolution product of (2.12) with $V$, observing (R1) as well as $V<V(0)<\infty$, one finds

$$
\rho * V \leqq V *|\Lambda|^{-1} \exp [\beta V(0)]
$$

and furthermore

$$
\left(V *|\Lambda|^{-1}\right)(\mathbf{r}) \leqq\left(V_{C} *|\Lambda|^{-1}\right)(\mathbf{r}) \leqq\left(V_{C} \bullet\left|B_{R^{\prime}}\right|^{-1}\right)(0)=K R^{2-d}
$$

for $K$ some fixed positive constant that depends on $h$. Recall that $\infty$ means convolution over $B_{R}$. Thus $\rho * V \rightarrow 0$ as $R \rightarrow \infty$. This estimate can be combined with Jensen's inequality to yield

$$
|\Lambda| \rho \leqq \exp (\rho * V-\llbracket \rho * V \rrbracket) \rightarrow 1
$$

as $R \rightarrow \infty$. Here, $\llbracket g \rrbracket$ is the homogeneous mean of $g$ over $\Lambda$. On the other hand, applying the above estimate for $\rho * V$ to the normalizing integral in (2.12) we find that on any $\Lambda^{\prime} \subset \Lambda(R)$

as $R \rightarrow \infty$.

$$
|\Lambda| \rho \geqq \exp \left\{-\beta \exp [\beta V(0)] K R^{2-d}\right\} \nearrow 1
$$

Remark 1. By Lemma 3.1 the homogeneity of the one-point density in the T-limit is recovered for the Gibbs states.

Remark 2. The recent review article by Bavaud [Bav] gives further results regarding the infinite-volume limit of similar equations. 
Lemma 3.1 leaves open what happens at $\beta=\infty$. For any finite $\Lambda$, i.e., not necessarily a ball, (2.12) converges to $\delta\left(\mathbf{r}-\mathbf{r}_{0}\right)$ as $\beta \rightarrow \infty$, with $\mathbf{r}_{0}$ some point in $\Lambda$ (see [Kie 2] for a proof). In this sense the delta function is a zero-temperature fixed point, and one may conjecture that the phase transition is shifted to zero temperature as $\Lambda \rightarrow \mathbb{R}^{d}$. We are now able to address the question of uniformity of the T-limit sequence. Theorem 3.1 summarizes what happens if one fixes $\beta$ and takes $\Lambda \rightarrow \mathbb{R}^{d}$ in the infinite-density ensemble.

Theorem 3.1. There exists at least one $\beta$ for which the infinite-density Gibbs states and corresponding mean thermodynamic functions of the finite-volume canonical ensemble undergo a phase transition when $R$ is varied for this value of $\beta$. Either the transition occurs at a discrete value $R_{\mathfrak{t}}(\beta)$ or for all $R$ in some interval there exists a line of multiple phases. On the other hand, if $\beta<\beta$, which defines the hightemperature regime, no phase transition occurs in the measures if $R$ is varied. $A$ discrete transition corresponds to a jump in the mean ideal energy and entropy.

Proof. Obvious.

Remark. Presumably a jump occurs in the pressure, counted per particle. The jump in the pressure means that the transition is anomalous.

Under the hypotheses that the Gibbs states, defined by the Gibbs postulate for the mean free energy on the space of infinite configurations, represent the physically relevant equilibrium states at sufficiently high density, and with the assumptions on the interaction $V$ as above, it follows that the T-limit sequence is nonuniform for at least one $\beta$. Presumable this is true for a whole interval of $\beta$ values beyond the critical $\not{\beta}$. Starting with a finite system in some finite domain at sufficiently low temperature in the collapsed state, passing over to bigger domains, keeping the temperature and the average density fixed (the usual T-limit sequence), results in a drastic change in the density profile to an almost homogeneous state in a region of big domains. Only after that happened the system settles down in accord with the principle of macroscopic equivalence and approaches the state predicted by the T-limit. This might have some consequences in situations in which (Coulomb) systems have extremely high density. Presumably such densities are only available in the interior of stars [Sha-Teu]. One can conceive of some internal collapse which may ignite some bigger spectacle.

Whether the Gibbs states are indeed the physically relevant states is, however, an open question so far. In the next sections we investigate the relation with the finite $N$ problem in some detail.

\section{The Limit $N \rightarrow \infty$ of the Thermodynamic Functions}

We now come to the discussion of the limit $N \rightarrow \infty, \Lambda$ fixed, of the finite- $N$ thermodynamic functions. The free energy of the measure (1.2) is

$$
F(N, \Lambda, \beta)=-\beta^{-1} \log \left[|\Lambda|^{-2 N} Q(N, \Lambda, \beta)\right],
$$

where $Q$ is given in (1.2b). The configurational energy is

$$
E(N, \Lambda, \beta)=\int_{\Lambda^{\times 2 N}} U^{(\mathbb{I})} \mu^{(\mathbb{I I})}(d X)=\partial_{\beta}[\beta F(N, \Lambda, \beta)] .
$$


The configurational entropy is given by

$$
S=-k_{B} \int_{A^{\times 2 N}} \log \left(|\Lambda|^{2 N} d \mu^{(\mathbb{I I})} / d X\right) \mu^{(\mathbb{I I})}(d X)=k_{B} \beta^{2} \partial_{\beta} F(N, \Lambda, \beta) .
$$

We first prove that the free energy per particle $(1 / 2 N) F(N, \Lambda, \beta)$ has a limit $f^{*}(\Lambda, \beta)$. For $\beta \searrow 0$, it is readily shown that $F \rightarrow-N \bar{V}$, where

$$
\bar{V} \equiv|\Lambda|^{-2} \int_{\Lambda^{\times 2}} V\left(\left|\mathbf{r}-\mathbf{r}^{\prime}\right|\right) d^{d} r d^{d} r^{\prime}
$$

and the existence of $\lim _{N \rightarrow \infty} F / 2 N$ is trivial for $\beta=0$. Let therefore $\beta>0$ from now on.

Proposition 4.1. For $\beta>0, \lim _{N \rightarrow \infty}(1 / 2 N) F(N, \Lambda, \beta) \equiv f^{*}(\Lambda, \beta)$ exists, with $f^{*}(\Lambda, \beta)=\inf _{N \in \mathbb{N}}(1 / 2 N) F(N, \Lambda, \beta)$. The mapping $\beta \mapsto \beta f^{*}(\Lambda, \beta)$ is strictly decreasing, concave, nonpositive, and right continuous on $[0, \infty)$. The mapping $\beta \mapsto f^{*}(\Lambda, \beta)$ is nonincreasing, negative, and left continuous on $(0, \infty]$.

Proof. The existence of a limit of $(1 / 2 N) F(N, \Lambda, \beta)$ follows from Griffiths' argument [Gri2, Sect. IIIB], which shows that the free energy of a chargesymmetric system is subadditive with respect to the number of particles. The free energy per particle is bounded below by $-B / 2$, by stability. It follows that $(1 / 2 N) F(N, \Lambda, \beta)$ converges to its infimum (e.g., [Ell, Lemma IX.2.4]). This concludes the existence part of the proof.

From $\partial_{\beta} E=E^{2}-\int U^{2} \mu^{(\mathbb{I I})}(d X) \leqq 0$ for $N<\infty, \quad$ and from $E(N, \Lambda, \beta) \stackrel{\beta \rightarrow 0}{\longrightarrow}-N \bar{V}<0$, it follows now with (4.2) that $\beta \mapsto \beta f^{*}$ is a limit of concave, strictly decreasing mappings on $[0, \infty)$. Concavity over $[0, \infty)$, continuity on $(0, \infty)$, and strict decrease on $[0, \infty)$ follow. This implies right continuity at $\beta=0$. Nonpositivity of $\beta \mapsto \beta f^{*}$ follows from the decrease and from $\left.\beta f^{*}\right|_{\beta=0}=0$. The latter follows from the Gibbs inequality for the canonical free energy [Gib, Ch. XI, Thm. III] with a homogeneous probability measure as trial measure. This gives $F(N, \Lambda, \beta) \leqq-N \bar{V}$. Together with $F / 2 N \geqq-B / 2$ it now follows that $\left.\beta F\right|_{\beta=0}=0$ for all $N$.

This also proves $F / 2 N<0$, hence $f^{*}<0$ strictly. Continuity of $\beta \mapsto f^{*}$ on $(0, \infty)$ is obvious. Left continuity at $\beta=\infty$ follows from the Gibbs inequality by choosing as trial measures products of identical measures with compact support a ball with radius shrinking $\sim \beta^{-1}$. As $\beta \rightarrow \infty$, the free energy per particle of such molified Dirac measures converges to $\min _{\beta} F / 2 N$, from which left continuity follows. Nonincrease of $\beta \mapsto f^{*}$ follows from the second equation in (4.3) and the fact that (4.3) takes its maximum value 0 .

As corollary follows:

Corollary 4.1. The limits $\lim _{N \rightarrow \infty}(1 / 2 N) E(N, \Lambda, \beta)$ and $\lim _{N \rightarrow \infty}(1 / 2 N) S(N, \Lambda, \beta)$ exist simultaneously except for an at most enumerable set of values of $\beta$. The limit functions, where they exists, are given by $e^{*}(\Lambda, \beta)=\partial_{\beta}\left[\beta f^{*}(\Lambda, \beta)\right]$ and $s^{*}(\Lambda, \beta)=k_{B} \beta^{2} \partial_{\beta} f^{*}(\Lambda, \beta)$. The mapping $\beta \mapsto e^{*}(\Lambda, \beta)$ is negative, nonincreasing and almost everywhere continuous. The mapping $\beta \mapsto s^{*}(\Lambda, \beta)$ is nonpositive, nonincreasing, and almost everywhere continuous. 
Proof. The claim follows from concavity of $\beta \mapsto \beta f^{*}$. See, for instance, [Gri 1, Appendix A].

The above results follow from standard arguments. What makes the problem challenging is the following.

Proposition 4.2. For any admissible $\Lambda$, the strict inequality $f^{*}<\inf _{\varrho \in \mathscr{M}_{+, 1}} \mathscr{F}(\rho)$ holds for all $\beta \in(0, \infty)$.

Proof. By Proposition 4.1 and Gibbs' variational principle,

$$
f^{*}(\Lambda, \beta) \leqq(1 / 2) F(1, \Lambda, \beta)=\inf _{\lambda} \varphi(\lambda)
$$

with $\lambda \in(\mathscr{M} \otimes \mathscr{M})_{+, 1}\left(\Lambda^{\times 2}\right)$ absolutely continuous, and

$$
\varphi(\lambda) \equiv-\frac{1}{2} \int_{\Lambda^{\times 2}}\left[V\left(\left|\mathbf{r}-\mathbf{r}^{\prime}\right|\right)-k_{B} T \log \left(|\Lambda|^{2} d \lambda / d^{2 d} r\right)\right] \lambda\left(d^{d} r d^{d} r^{\prime}\right)
$$

the Gibbs free-energy functional per particle for $N=1$ (one pair). The unique global minimizer $\lambda^{*}$ of $\varphi$ is given by $\lambda^{*}=\mu^{(N=1)}$. Obviously, $\mu^{(1)}$ is not a product measure. Since $\left.\varphi(\lambda)\right|_{\lambda=\varrho \otimes \varrho}=\mathscr{F}(\rho)$ for absolutely continuous $\varrho \in \mathscr{M}_{+, 1}(\Lambda)$, Proposition 4.2 is proved.

The procedures of taking the limit of infinitely many variables and of minimizing the free energy obviously are noninterchangeable at the level of the thermodynamic functions. The differences are rooted in the correlations of the finite- $N$ systems, into which we have to inquire next.

\section{Some Results about Correlations}

Let us begin with a discussion of the energy functional. The energy (4.2) of a system of $2 N$ particles can be rewritten in terms of expectations taken with the second marginals (both neutral and non-neutral) of $\mu^{(N)}$. It becomes a sum of a term proportional to $N$ and another one proportional to $N^{2}$.

$$
\begin{aligned}
E(N, \Lambda, \beta)= & -N \int_{\Lambda_{\times 2}} V\left(\left|\mathbf{r}-\mathbf{r}^{\prime}\right|\right) \mu_{2 \mid 2}^{(N)}\left(d^{d} r d^{d} r^{\prime}\right) \\
& +N^{2} \int_{\Lambda^{\times 2}} V\left(\left|\mathbf{r}-\mathbf{r}^{\prime}\right|\right)\left[\mu_{2 \mid 2}^{(N)}-\mu_{2 \mid 0}^{(N)}\right]\left(d^{d} r d^{d} r^{\prime}\right) .
\end{aligned}
$$

(The appearance of $\mu_{2 \mid 2}^{(N)}$ is of course by choice, since $\mu_{2 \mid 2}^{(N)}=\mu_{2 \mid-2}^{(N)}$.) Let the first integral in (5.1) be abbreviated by

$$
-\frac{1}{2} \int_{\Lambda^{\times 2}} V\left(\left|\mathbf{r}-\mathbf{r}^{\prime}\right|\right) \mu_{2 \mid 2}^{(N)}\left(d^{d} r d^{d} r^{\prime}\right) \equiv e^{(N)}
$$

and the second one by

$$
\frac{1}{2} N \int_{\Lambda^{\times 2}} V\left(\left|\mathbf{r}-\mathbf{r}^{\prime}\right|\right)\left[\mu_{2 \mid 2}^{(N)}-\mu_{2 \mid 0}^{(N)}\right]\left(d^{d} r d^{d} r^{\prime}\right) \equiv \varepsilon^{(N)} .
$$

To discuss their behavior as $N \rightarrow \infty$ one needs the following lemma. 
Lemma 5.1. The decomposition measure $\tilde{v}$ of any weak limit point $\mu$ of $\left\{\mu^{(N)}\right\}$ is concentrated on the charge-symmetric product measures $\varrho^{\otimes \mathbb{N}}$.

Proof. If $\mu^{(N[k])} \rightarrow \mu$ for a subsequence $N(k) \rightarrow \infty$, then

$$
\mu=\int \tilde{v}_{\mu}\left(d \varrho_{1} \otimes d \varrho_{2}\right) \varrho_{1}^{\otimes 2 \mathbb{N}} \otimes \varrho_{2}^{\otimes 2 \mathbb{N}-1} .
$$

By (2.2) one finds

$$
\int V \mu_{2 \mid 2}=\int \tilde{v}_{\mu}\left(d \varrho_{1} \otimes d \varrho_{2}\right) \frac{1}{2}\left[\int V \varrho_{1}^{\otimes 2}+\int V \varrho_{2}^{\otimes 2}\right] \geqq 0
$$

which together with $V \leqq B$ implies

$$
0 \geqq \lim _{k \rightarrow \infty} e^{(N[k])} \geqq-\frac{1}{2} V(0) \text {. }
$$

This and $-\bar{V} \geqq N^{-1} E(N, \Lambda, \beta) \geqq-V(0)$ implies that $N^{-1} \varepsilon^{(N)} \rightarrow 0$ for $N \rightarrow \infty$. After rewriting $\mu$ with the aid of the representation (2.1), we find

$$
\int V\left[\mu_{2 \mid 2}-\mu_{2 \mid 0}\right]=\frac{1}{2} \int \tilde{v}_{\mu}\left(d \varrho_{1} \otimes d \varrho_{2}\right) \int V\left(\varrho_{1}-\varrho_{2}\right)^{\otimes 2}
$$

Since $V$ is of positive type, it follows that $\int V\left[\mu_{2 \mid 2}-\mu_{2 \mid 0}\right] \geqq 0$ with equality holding only for $\tilde{v}$ concentrated on charge-symmetric products.

This establishes local charge neutrality of the weak limit points of the measures. The analog for the Gibbs states on the infinite configurations was proved in Sect. 2.

An immediate consequence of Lemma 5.1 is

Corollary 5.1. If $\mu^{(N[k])} \rightarrow \mu$ for a subsequence $N(k) \rightarrow \infty$, then $e^{(N[k])} \rightarrow e(\mu)$.

Proof. Follows from the definition of weak convergence.

Since the correlations contribute in the limit to the energy, this must happen through the integral (5.3). By Corollaries 4.1 and 5.1 it follows that, given $\beta$ and $\Lambda$, for any subsequence $\{N[k] \mid k \in \mathbb{N}\}$ such that $(2 N[k])^{-1} E(N[k], \Lambda, \beta) \rightarrow e^{*}$ and $\mu^{(N[k])} \rightarrow \mu$, there exists a limit

$$
\lim _{k \rightarrow \infty} \varepsilon^{(N[k])}=\varepsilon_{(e ; \mu)} .
$$

Since for almost all $\beta$ the limit of $(2 N)^{-1} E(N, \Lambda, \beta)$ exists, the index $(e ; \mu)$ can be replaced by a single index $(\mu)$ for almost all $\beta$.

Proposition 5.1. Any limit point of the correlation energy per particle is strictly negative, i.e., $\varepsilon_{(e ; \mu)}<0$.

In order to prove Proposition 5.1 one needs a bound that relates the limit points of the entropy per particle to the mean entropy of the corresponding weak limit points $\mu$ of the measures. The definition of an entropy of a neutral marginal $\mu_{2 n \mid 0}^{(N)}$ is analogous to $(2.3 \mathrm{~b})$,

$$
S_{\mu^{(N)}}(\mathbf{N})=-k_{B} \int_{\Lambda^{\times 2 n}} \eta_{2 n \mid 0}^{(N)} \log \left(|\Lambda|^{2 n} \eta_{2 n \mid 0}^{(N)}\right) \prod_{i \in \mathbf{N}} d^{d} r_{i}
$$

where $\eta_{2 n \mid 0}^{(N)}$ is the density (with respect to Lebesgue measure) of $\mu_{2 n \mid 0}^{(N)}$. 
Lemma 5.2. Let $\beta$ be given, and let $\lim _{k \rightarrow \infty}(2 N[k])^{-1} S(N[k], \Lambda, \beta)$ exist. Then the mean entropy of any $\mu \in \operatorname{limpt}\left\{\mu^{(N[k])}\right\}$ obeys the inequality

$$
\lim _{k \rightarrow \infty}(2 N[k])^{-1} S(N[k], \Lambda, \beta) \leqq s(\mu) .
$$

Proof of Lemma 5.2. Let II and $\mathbf{N}$ be finite sets, with $|\mathbb{I}|=2 N>2 n=|\mathbf{N}|$. Let $\{\mathbf{N}\}$ be the set of sets with cardinality $2 n$, and let $\mathbf{M}=\bigcup_{i=1}^{m} \mathbf{N}_{i}$ be a union of $m$ pairwise disjoint elements of $\{\mathbf{N}\}$ which is contained in II, with $m(N, n)$ the greatest possible number. Finally, pick a subsequence $\left\{N^{\prime}[k]\right\} \subset\{N[k]\}$ such that $\mu^{\left(N^{\prime}[k]\right)} \rightarrow \mu$.

An analog of Proposition 2.2 holds for (5.4) as well and yields, with $\mathbb{I}=\mathbf{M} \cup(\mathbb{I} \backslash \mathbf{M})$,

$$
S(N, \Lambda, \beta)=S_{\mu^{(N)}}(\mathbb{I}) \leqq m(N, n) S_{\mu^{(N)}}(\mathbf{N})+S_{\mu^{(N)}}(\mathbb{I} \backslash \mathbf{M}) \leqq m(N, n) S_{\mu^{(N)}}(\mathbf{N})
$$

(sub-additivity of the entropy and permutation invariance of $\mu^{(N)}$ giving the first inequality; non-positivity the second). With $m(N, n) / N>1 /[n+n / m(N, n)] \rightarrow 1 / n$, and noting upper semi-continuity of the entropy functional, taking limits now gives

$$
\begin{aligned}
\lim _{k \rightarrow \infty}(2 N[k])^{-1} S(N[k], \Lambda, \beta) & =\lim _{k \rightarrow \infty}\left(2 N^{\prime}[k]\right)^{-1} S\left(N^{\prime}[k], \Lambda, \beta\right) \\
& \leqq \liminf _{k}(1 / 2 n) S_{\mu^{\left(N^{\prime}[k]\right)}}(\mathbf{N}) \\
& \leqq \lim _{k} \sup (1 / 2 n) S_{\mu^{\left(N^{\prime}[k]\right)}}(\mathbf{N}) \\
& \leqq(1 / 2 n) S_{\mu}(\mathbf{N})
\end{aligned}
$$

for all $\mathbf{N}$. Thus

$$
\lim _{k \rightarrow \infty}(2 N[k])^{-1} S(N[k], \Lambda, \beta) \leqq s(\mu)
$$

for any $\mu \in \operatorname{limpt}\left\{\mu^{(N[k])}\right\}$.

What we need below is a weaker form of Lemma 5.2,

Corollary 5.2. For given $\beta$ and $\Lambda$,

Proof. Trivial.

$$
\lim \sup (2 N)^{-1} S(N, \Lambda, \beta) \leqq \sup _{\mu \in \operatorname{limpt}\left\{\mu^{(N)}\right\}} S(\mu) .
$$

Remark. The $\overline{\lim }(1 / 2 N) S(N$, .) always exists, which follows from the sandwich bounds for the free energy and for the energy (see [Kie 1]). For almost all $\beta$ this lim sup is actually a limit; see Corollary 4.1.

Proof of Proposition 5.1. To show that any limit point of $\left\{\varepsilon^{(N)}\right\}$ is negative, assume that $\limsup _{N \rightarrow \infty} \varepsilon^{(N)} \geqq 0$. In that case there exists a subsequence $\{N[k]\}$ such that $\mu^{(N[k])} \stackrel{N \rightarrow \infty}{\rightarrow} \mu$ and $(1 / 2 N[k]) E(N[k], \Lambda, \beta) \rightarrow e^{*}$, and the inequality

$$
\lim _{k \rightarrow \infty}(2 N[k])^{-1} E(N[k], \Lambda, \beta) \geqq e(\mu) \geqq \inf _{\mu^{\prime} \in \operatorname{limpt}\left\{\mu^{(N)}\right\}} e\left(\mu^{\prime}\right)
$$

holds. Combining this with Corollary 5.2, and using the equality $F(N, \Lambda, \beta)$ $=E(N, \Lambda, \beta)-T S(N, \Lambda, \beta)$, one gets 


$$
f^{*}=\lim _{k \rightarrow \infty}(2 N[k])^{-1} F(N[k], \Lambda, \beta) \geqq \inf _{\mu \in \operatorname{limpt}\left\{\mu^{(N)}\right\}} f(\mu) \geqq \inf _{\mu \in \mathscr{P}} f(\mu)=\inf _{\rho} \mathscr{F}(\rho)
$$

in contradiction to Proposition 4.2. Consequently,

$$
\lim _{k \rightarrow \infty}(2 N[k])^{-1} E(N[k], \Lambda, \beta)<e(\mu)
$$

strictly for any convergent subsequence.

The physical picture (usually stated in plasma physics textbooks) that a charged particle is on the average closer to particles of oppositely signed charge than to particles of the same sign is recovered in the high-density limit at all finite temperatures and for all two-component systems with positive type interactions as described in the introduction. The contribution of the correlations to the total energy integral produces negative limit points.

However, the limit of (5.3) cannot be written as a functional of the limit measures, which is manifest from the proof of Lemma 5.1. In so far the correlation energy only contributes a shift to the standard Definition 3 of the mean free energy. It is nevertheless conceivable that, although all limit points of the measures are superpositions of locally charge-neutral correlation-free states, the finite- $N$ correlations may be "visible" in the sense that they may forbid certain of the correlation-free states. In particular, this means we need to take the results of Sect. 2 and 3 with a grain of salt since the Gibbs hypothesis for the states on the space of infinite configurations need not yield the weak limit points of the finite- $N$ measures. One or the other surprise may be waiting here.

Acknowledgement. Special thanks go to H. Spohn for very helpful discussions and comments. In the course of the development of this work I also received useful comments and criticism from many individuals, in particular M. Aizenman, A. Compagner, G.A. Goldin, B. Jancovici, E. H. Lieb, H. Narnhofer, C. Newman, J. Percus, L. Sadun, K. Schindler, A. Sokal, H.T. Yau, and H. J. Ziegler. I would further like to thank H. Weitzner for encouragement and W. S. Lawson for commenting on the manuscript. This work was supported by the U.S. Department of Energy under grant DE-FG02-86ER 53223.

\section{References}

[Ama] Amann, H.: Fixed point equations and nonlinear eigenvalue problems in ordered

Banach spaces. SIAM Rev. 18, 620 (1976)

[Bal] Balescu, R.: Equilibrium and nonequilibrium statistical mechanics. New York: Wiley 1975

[Bav] Bavaud, F.: Equilibrium properties of the Vlasov functional: the generalized Poisson-Boltzmann-Emden equation. Rev. Mod. Phys. 63, 129 (1991)

[Cam-O’N] Campbell L.J., O’Neil, K.: Statistics of 2-d point vortices and high energy vortex states. Los Alamos Preprint LA-UR 90-3960, to appear in J. Stat. Phys. (1991)

[Com] Compagner, A.: Thermodynamics as the continuum limit of statistical mechanics. Am. J. Phys. 57, 106 (1989)

[C-B-R] Compagner, A., Bruin, C., Roelse, A.: Collapsing systems. Phys. Rev. A 39, 5989 (1989)

[E11] Ellis, R.S.: Entropy, large deviations, and statistical mechanics. Berlin, Heidelberg, New York: Springer 1985 
[Fis] Fisher, M.E.: The free energy of a macroscopic system. Arch. Rat. Mech. Anal. 17, 377 (1967)

[Fis-Rue] Fisher, M.E., Ruelle, D.: The stability of many-particle systems. J. Math. Phys. 7, $260(1966)$

[Frö-Par 1] Fröhlich, J., Park, Y.M.: Correlation inequalities and the thermodynamic limit for classical and quantum continuous systems. Commun. Math. Phys. 59, 235 (1978)

[Frö-Par 2] Fröhlich, J., Park, Y.M.: Correlation inequalities and the thermodynamic limit for classical and quantum continuous systems. II. Bose-Einstein and Fermi-Dirac statistics. J. Stat. Phys. 23, 701 (1980)

[Frö-Rue] Fröhlich, J., Ruelle, D.: Statistical mechanics of vortices in an inviscid twodimensional fluid. Commun. Math. Phys. 87, 1 (1982)

[Gib] Gibbs, J. W.: Elementary principles of statistical mechanics. In: The Collected Works of Gibbs, J.W., Vol. II. New York: Longmans 1931

[Gri1] Griffiths, R. B.: A proof that the free energy of a spin system is extensive. J. Math. Phys. 5, 1215 (1964)

[Gri 2] Griffiths, R. B.: Free energy of interacting magnetic dipoles. Phys. Rev. 176, 655 (1968)

[Her-Thi 1] Hertel, P., Thirring, W.: Free energy of gravitating Fermions. Commun. Math. Phys. 24, 22 (1971)

[Her-Thi2] Hertel, P., Thirring, W.: Thermodynamic instability of a system of gravitating Fermions. In: Quanten und Felder. Dürr, H.P., (ed.). Braunschweig: Vieweg 1971

[H-N-T] Hertel, P., Narnhofer, H., Thirring, W.: Thermodynamic functions for Fermions with gravostatic and electrostatic interactions. Commun. Math. Phys. 28, 159 (1972)

[Hew-Sav] Hewitt, E., Savage, L. J.: Symmetric measures on Cartesian products. Trans. Am. Math. Soc. 80, 470, (1955)

[Kie1] Kiessling, M.K.-H.: A complementary thermodynamic limit for classical Coulomb matter. J. Stat. Phys. 59, 1157 (1990)

[Kie 2] Kiessling, M.K.-H.: On the equilibrium statistical mechanics of isothermal classical self-gravitating matter. J. Stat. Phys. 55, 203 (1989)

[Kie 3] Kiessling, M.K.-H.: Statistical mechanics of classical particles with logarithmic interactions. To appear in Commun. Pure Appl. Math. (1992)

[Lee-Yan] Lee, T. D., Yang, C. N.: Statistical theory of equations of state and phase transitions. II. Lattice gas and Ising model. Phys. Rev. 87, 410 (1952)

[Lie-Leb] Lieb, E. H., Lebowitz, J.L.: The constitution of matter: existence of thermodynamics for systems composed of electrons and nuclei. Adv. Math. 9, 316 (1972)

[Mes 1] Messer, J.: The pressure of Fermions with gravitational interactions. Z. Phys. 33, 313 (1979)

[Mes 2] Messer, J.: Temperature dependent Thomas-Fermi theory. Lecture Notes in Physics No. 147. Berlin, Heidelberg, New York: Springer 1981

[Mes 3] Messer, J.: On the gravitational phase transition in the Thomas-Fermi model. J. Math. Phys. 22, 2910 (1981)

[Mes 4] Messer, J.: Non-monotonicity of the mass distribution and the existence of the gravitational phase transition. Phys. Lett. 83A, 304 (1981)

[Mes-Spo] Messer, J., Spohn, H.: Statistical mechanics of the isothermal Lane-Emden equation. J. Stat. Phys. 29, 561 (1982)

[Pfl] Pflug, A.: Gravitating Fermions in an infinite configuration space. Commun. Math. Phys. 78, 83 (1980)

[P-N-T] Posch, H. A., Narnhofer, H., Thirring, W.: Dynamics of unstable systems. Phys. Rev. A 42, $1880(1990)$

[Ree-Sim] Reed, M., Simon, B.: Functional analysis. New York: Academic Press 1980

[Rob-Rue] Robinson, D.W., Ruelle, D.: Mean entropy of states in classical statistical mechanics. Commun. Math. Phys. 5, 288 (1967)

[Rue 1] Ruelle, D.: Statistical mechanics. New York: Benjamin 1969

[Rue 2] Ruelle, D.: Classical statistical mechanics of a system of particles. Helv. Phys. Acta 36, 183 (1963)

[Rue 3] Ruelle, D.: Statistical mechanics of quantum systems of particles. Helv. Phys. Acta 36, 789 (1963) 
[Sha-Teu] Shapiro, S., Teukolsky, S.: Black holes, white dwarfs and neutron stars. New York: Wiley 1983

[Thi] Thirring, W.: Lehrbuch der mathematischen Physik 4. Vienna: Springer 1980

[vHo] van Hove, L.: Quelques propriétés générales de l'intégrale de configuration d'un système de particule avec interaction. Physica 15, 951 (1949)

[Yan-Lee] Yang, C.N., Lee, T. D.: Statistical theory of equations of state and phase transitions. I. Theory of condensation. Phys. Rev. 87, 404 (1952)

Communicated by M. Aizenman 
